RESEARCH BRIEF

\title{
Summer Weight Gain Among Preschool- Aged Children With Obesity: An Observational Study in Head Start
}

\author{
Jacob P. Beckerman-Hsu, $\mathrm{PhD}^{1}$; Alyssa Aftosmes-Tobio, $\mathrm{MPH}^{1}$; Kindra Lansburg, $\mathrm{MPH}^{2}$; \\ Jessie Leonard, JD ${ }^{3}$; Merieka Torrico, $\mathrm{MPH}^{2}$; Erica L. Kenney, $\mathrm{ScD}^{4}$; \\ SV Subramanian, $\mathrm{PhD}^{5,6}$; Sebastien Haneuse, $\mathrm{PhD}^{7}$; Kirsten K. Davison, $\mathrm{PhD}^{1}$
}

\begin{abstract}
Accessible Version: www.cdc.gov/pcd/issues/2021/20_0532.htm
Suggested citation for this article: Beckerman-Hsu JP, AftosmesTobio A, Lansburg K, Leonard J, Torrico M, Kenney EL, et al. Summer Weight Gain Among Preschool-Aged Children With Obesity: An Observational Study in Head Start. Prev Chronic Dis 2021;18:200532. DOI: https://doi.org/10.5888/pcd18.200532.
\end{abstract}

\section{PEER REVIEWED}

\section{Summary}

What is already known on this topic?

School-aged children gain weight more rapidly in the summer than during the academic year. The limited evidence on summer weight gain among preschool-aged children is mixed.

What is added by this report?

We found that preschool-aged children with obesity in Head Start experienced significant summer weight gain.

What are the implications for public health practice?

As with school-aged children, increased focus on the summer is warranted for promoting healthy weight among children in Head Start.

\section{Abstract}

School-aged children gain weight most rapidly in summer, but few studies have investigated summer weight gain among preschoolaged children. We fit continuous linear spline mixed models to test for accelerated summer weight gain among 2,044 children attending 16 Boston-area Head Start programs between fall 2016 and spring 2019. Academic year and summer rates of change in modified body mass index $z$-score differed $(P<.001)$, with accelerated summer weight gain most pronounced among children with obesity. As with school-aged children, increased focus on the sum- mer is warranted for promoting healthy weight among children in Head Start.

\section{Objective}

School-aged children experience more rapid weight gain during summer than the academic year (1). Previous studies on summer weight gain among preschoolers have yielded mixed results (2-4). Having a better understanding of when preschoolers gain excess weight is important; $13.7 \%$ of 2- to 5-year-old children in the United States already have obesity (5), putting them at increased risk for obesity and adverse health outcomes at later ages (6). In our study, we used longitudinal height and weight data from more than 2,000 children to test the hypothesis that preschool-aged children enrolled in Head Start experience accelerated summer weight gain.

\section{Methods}

\section{Sample}

From fall 2016 through fall 2018, 2,539 children enrolled in 16 Head Start programs serving Boston, Cambridge, and Somerville, Massachusetts. The 2,044 children we included in the main analysis for this observational, longitudinal study had a valid body mass index (BMI) measurement in their first fall of Head Start. BMI data cleaning protocols removed $6.2 \%$ of BMI measurements (supplemental information is available at https:// dataverse.harvard.edu/dataset.xhtml?persistentId=doi:10.7910/ DVN/EXMNNM).

Sample characteristics extracted from Head Start administrative records include child age, sex, race/ethnicity (Hispanic/Latino, non-Hispanic Asian, non-Hispanic Black or African American, non-Hispanic White, and non-Hispanic other or multiracial), and calendar year of Head Start entry, as well as parent age, sex, edu- 
cation (less than high school, high school graduate, greater than high school), and family structure (single parent or 2 parents). We used maternal characteristics when available; more than $96 \%$ of parents in the sample identified as mothers or mother figures.

\section{Modified BMI z-score}

Each fall and spring, trained Head Start staff measure children's height and weight by using standardized equipment provided for an ongoing obesity prevention trial (7). We used Centers for Disease Control and Prevention 2000 reference curves (8) to calculate age- and sex-specific weight status (underweight, healthy weight, overweight, obese).

As suggested elsewhere (9), we used modified BMI z-scores (mB$\mathrm{MIz}$ ) as the study outcome; $\mathrm{mBMIz}$ is interpreted similarly to standard BMI z-scores (BMIz), with 0 indicating the median ageand sex-specific BMI. mBMIz is more appropriate for longitudinal analysis. Unlike BMIz, it is sensitive to change even for children with high BMIs. (Appendix 2 of the supplemental information contains more detail on mBMIz.)

\section{Analysis}

We summarized sample characteristics at enrollment. We used continuous linear spline mixed models to estimate the rate of change in mBMIz during academic year 1, the summer, and academic year 2 by initial weight status. Random program-level intercepts, child-level intercepts, and child-level slopes were included to account for repeated measurements on children, who were nested in Head Start programs. (Appendix 3 of the supplemental information contains model specification details.) We fit models with maximum likelihood and used likelihood ratio tests for the null hypothesis that the rate of change in mBMIz was the same across year 1, summer, and year 2 of Head Start. The main analysis was unadjusted because initial weight status was weakly associated with the number of BMI measurements taken $(P=.64)$. Alpha was set at .05 a priori. All analysis was completed in SAS version 9.4 (SAS Institute Inc). Institutional review board approval was granted by the Harvard T.H. Chan School of Public Health and Boston College.

\section{Results}

In fall of their first year in Head Start, children were aged 29 to 47 months (Table). Most were Hispanic/Latino (45.5\%) or nonHispanic Black/African American (34.1\%), and the prevalence of obesity was $18.4 \%$.

Overall, mBMIz trajectory changed significantly with the onset of summer (Figure, $P<.001$ ). Accelerated summer weight gain was most pronounced among children with obesity, who had an aver- age change in mBMIz of -0.30 /y during academic year 1 , followed by a significant increase in rate of change to $1.05 \mathrm{mBMIz} / \mathrm{y}$ over the summer $(P<.001)$, and a subsequent significant decrease in rate of change to $-0.13 \mathrm{mBMIz} / \mathrm{y}$ during academic year $2(P<$ $.001)$. These results were robust to multivariable adjustment and stricter sample inclusion criteria (more information is available at ht t p s://datavers e.harvard.edu/dataset.xhtm l? persistentId=doi:10.7910/DVN/EXMNNM). Accelerated summer weight gain was also observed among children with overweight, although the pattern was less pronounced and was not significant across all sensitivity analyses.

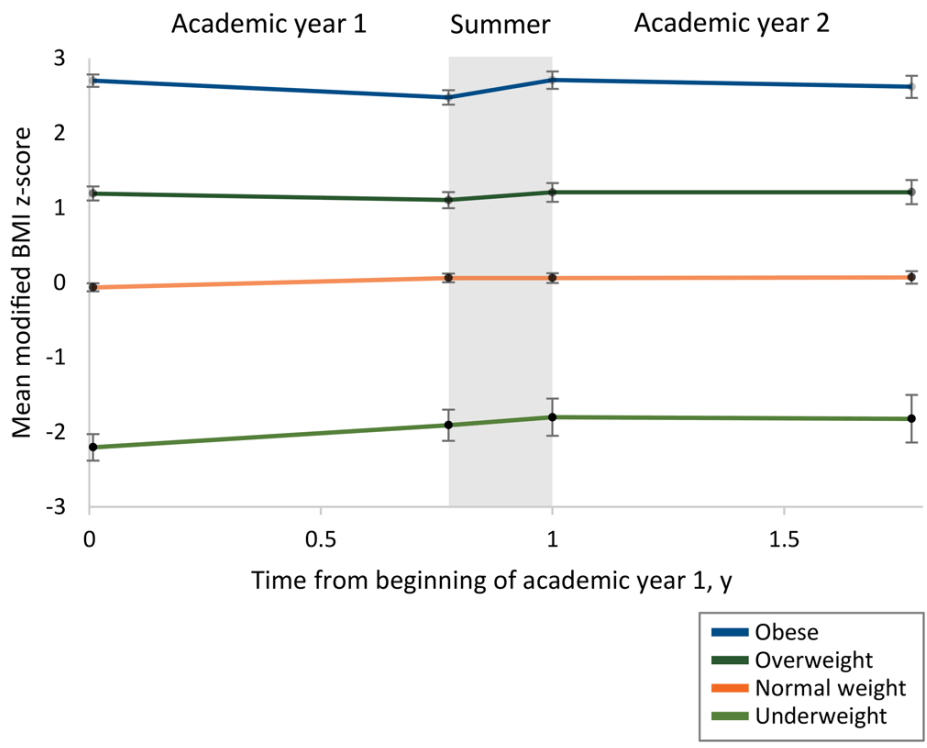

Figure. Mean modified body mass index (BMI) z-score among children who were underweight, healthy weight, overweight, and obese at enrollment in Head Start. Data are from children enrolled in Head Start in Boston, Cambridge, and Somerville, Massachusetts, 2016-2019. Estimates are from a single mixed-model with random program-level intercepts and random childlevel intercepts and slopes. Error bars show 95\% confidence intervals at the beginning of academic year 1 , the end of academic year 1 , the beginning of academic year 2 , and the end of academic year 2 . Across all children, mean modified BMI Z-score trajectory changed significantly upon the onset of summer $\left(X^{2}=108.8, d f=15, P<.001\right)$.

\section{Discussion}

Children in Head Start have distinct academic year and summer rates of change in mBMIz, and children with obesity experienced significantly accelerated summer weight gain. Similar patterns have been observed among school-aged children (1) and in 2 previous studies of preschoolers $(2,4)$, adding to the evidence that efforts to promote healthy child weight may be most needed in summer. The lack of a structured daily schedule (10), the biological re-

The opinions expressed by authors contributing to this journal do not necessarily reflect the opinions of the U.S. Department of Health and Human Services, the Public Health Service, the Centers for Disease Control and Prevention, or the authors' affiliated institutions. 
sponse to longer day length (11), or both may explain accelerated summer weight gain. However, more research is necessary among preschool-aged children, because another study found a decrease in BMIz over the summer among preschool-aged children with obesity (3).

Our study has many strengths that make it an important contribution to the literature. We used administrative BMI data for a large sample of preschool-aged children. Height and weight measurements were taken by trained Head Start staff members who used standardized equipment, and we used mBMIz for both longitudinal data cleaning and modeling. Previous studies that used BMIz rather than $\mathrm{mBMIz}$ may have underestimated the magnitude of changes for children with high BMI (9).

A potential limitation to this study is that, as in previous Head Start studies, many children had less than the maximum of 4 BMI measurements $(2,3)$. We anticipate the magnitude of any selection bias induced by missing data to be small because weight status at enrollment was not significantly associated with the number of BMI measurements taken. Another limitation of this study is its generalizability, which was restricted to low-income children participating in Head Start. Patterns of weight gain may differ for higher-income preschool-aged children and for children in other early care and education settings, especially those not in centerbased care during the academic year.

We found that preschool-aged children in Head Start with obesity experienced accelerated summer weight gain. Like school-aged children, increased focus on the summer is warranted for promoting healthy weight among children in Head Start.

\section{Acknowledgments}

This work was supported by the National Institutes of Health (grant no. R01DK108200, K.K.D. principal investigator). No funding agency was involved in the study design, data collection, data analysis, writing of the manuscript, or decision to submit the manuscript for publication. No copyrighted materials were used in this article.

\section{Author Information}

Corresponding Author: Jacob P. Beckerman-Hsu, Boston College School of Social Work, McGuinn Hall 106K, 140 Commonwealth Ave, Chestnut Hill, MA 02467. Email: jacob.beckerman@bc.edu.

Author Affiliations: ${ }^{1}$ Boston College School of Social Work, Chestnut Hill, Massachusetts. ${ }^{2}$ Action for Boston Community Development, Boston, Massachusetts. ${ }^{3}$ Community Action Agency of Somerville, Somerville, Massachusetts. ${ }^{4}$ Department of
Nutrition and Department of Social and Behavioral Sciences, Harvard T.H. Chan School of Public Health, Boston, Massachusetts. ${ }^{5}$ Department of Social and Behavioral Sciences, Harvard T.H. Chan School of Public Health, Boston, Massachusetts. ${ }^{6}$ Harvard Center for Population and Development Studies, Cambridge, Massachusetts. ${ }^{7}$ Department of Biostatistics, Harvard T.H. Chan School of Public Health, Boston, Massachusetts.

\section{References}

1. Tanskey LA, Goldberg J, Chui K, Must A, Sacheck J. The state of the summer: a review of child summer weight gain and efforts to prevent it. Curr Obes Rep 2018;7(2):112-21.

2. Lumeng JC, Kaciroti N, Frisvold DE. Changes in body mass index $\mathrm{z}$ score over the course of the academic year among children attending Head Start. Acad Pediatr 2010; 10(3):179-86.

3. Lumeng JC, Kaciroti N, Sturza J, Krusky AM, Miller AL, Peterson KE, et al. Changes in body mass index associated with head start participation. Pediatrics 2015;135(2):e449-56.

4. Isojima T, Kato N, Yokoya S, Ono A, Tanaka T, Yokomichi $\mathrm{H}$, et al. Early excessive growth with distinct seasonality in preschool obesity. Arch Dis Child 2019;104(1):53-7.

5. Skinner AC, Ravanbakht SN, Skelton JA, Perrin EM, Armstrong SC. Prevalence of obesity and severe obesity in US children, 1999-2016. Pediatrics 2018;141(3):e20173459.

6. Ward ZJ, Long MW, Resch SC, Giles CM, Cradock AL, Gortmaker SL. Simulation of growth trajectories of childhood obesity into adulthood. N Engl J Med 2017;377(22):2145-53.

7. Beckerman JP, Aftosmes-Tobio A, Kitos N, Jurkowski JM, Lansburg K, Kazik C, et al.; CHL study team. Electronic address: chlheadstart@gmail.com. Communities for healthy living ( $\mathrm{CHL})$ - A family-centered childhood obesity prevention program integrated into Head Start services: study protocol for a pragmatic cluster randomized trial. Contemp Clin Trials 2019;78:34-45.

8. Kuczmarski RJ, Ogden CL, Guo SS, Grummer-Strawn LM, Flegal KM, Mei Z, et al.2000 CDC Growth Charts for the United States: methods and development. Vital Health Stat 11 2002;(246):1-190.

9. Chambers M, Tanamas SK, Clark EJ, Dunnigan DL, Kapadia CR, Hanson RL, et al. Growth tracking in severely obese or underweight children. Pediatrics 2017;140(6):e20172248.

10. Brazendale K, Beets MW, Weaver RG, Pate RR, TurnerMcGrievy GM, Kaczynski AT, et al. Understanding differences between summer vs. school obesogenic behaviors of children: the structured days hypothesis. Int J Behav Nutr Phys Act 2017;14(1):100.

The opinions expressed by authors contributing to this journal do not necessarily reflect the opinions of the U.S. Department of Health and Human Services, the Public Health Service, the Centers for Disease Control and Prevention, or the authors' affiliated institutions. 
11. Moreno JP, Crowley SJ, Alfano CA, Thompson D. Physiological mechanisms underlying children's circannual growth patterns and their contributions to the obesity epidemic in elementary school age children. Obes Rev 2020; 21(3):e12973. 


\section{Table}

Table. Characteristics of Children Enrolled in Head Start and Their Parents, Boston, Cambridge, and Somerville, Massachusetts, 2016-2019 ${ }^{a}$

\begin{tabular}{|c|c|c|c|c|c|c|}
\hline \multirow[b]{2}{*}{ Characteristic } & \multicolumn{4}{|c|}{ Number of BMI measurements } & \multirow{2}{*}{$\begin{array}{c}\text { Total } \\
(\mathrm{N}=2,044)\end{array}$} & \multirow[b]{2}{*}{$P^{\mathrm{a}}$} \\
\hline & $1(N=266)$ & $2(N=953)$ & $3(N=199)$ & $4(N=626)$ & & \\
\hline \multicolumn{7}{|c|}{ Children } \\
\hline \multicolumn{7}{|l|}{ Age, mo } \\
\hline 29-33 & 13.9 & 10.9 & 13.6 & 13.4 & 12.3 & \multirow{4}{*}{$<.001$} \\
\hline $34-38$ & 42.9 & 35.9 & 38.2 & 39.0 & 38.0 & \\
\hline $39-43$ & 34.6 & 42.8 & 30.7 & 28.0 & 36.0 & \\
\hline $44-47$ & 8.7 & 10.4 & 17.6 & 19.7 & 13.7 & \\
\hline \multicolumn{7}{|l|}{ Sex } \\
\hline Male & 50.4 & 51.5 & 51.3 & 47.4 & 50.1 & \multirow{2}{*}{.45} \\
\hline Female & 49.6 & 48.5 & 48.7 & 52.6 & 49.9 & \\
\hline \multicolumn{7}{|l|}{ Race/ethnicity (missing, $n=8$ ) } \\
\hline Non-Hispanic Asian & 7.2 & 9.7 & 9.1 & 12.6 & 10.2 & \multirow{5}{*}{.04} \\
\hline Non-Hispanic Black/African American & 31.4 & 33.7 & 31.2 & 37.0 & 34.1 & \\
\hline Hispanic/Latino & 52.3 & 46.8 & 45.2 & 40.6 & 45.5 & \\
\hline Non-Hispanic other or multi-racial & 2.7 & 3.6 & 5.0 & 4.5 & 3.9 & \\
\hline Non-Hispanic White & 6.4 & 6.2 & 9.6 & 5.3 & 6.3 & \\
\hline \multicolumn{7}{|l|}{ Calendar year of Head Start entry } \\
\hline 2016 & 31.6 & 29.5 & 41.2 & 50.6 & 37.4 & \multirow{3}{*}{$<.001$} \\
\hline 2017 & 25.2 & 24.6 & 58.8 & 49.4 & 35.6 & \\
\hline 2018 & 43.2 & 46.0 & 0.0 & 0.0 & 27.1 & \\
\hline \multicolumn{7}{|l|}{ Initial weight status } \\
\hline Underweight & 6.4 & 3.3 & 4.0 & 3.8 & 3.9 & \multirow{4}{*}{.64} \\
\hline Healthy weight & 60.5 & 62.6 & 64.8 & 63.1 & 62.7 & \\
\hline Overweight & 14.3 & 14.9 & 14.6 & 15.7 & 15.0 & \\
\hline Obese & 18.8 & 19.2 & 16.6 & 17.4 & 18.4 & \\
\hline \multicolumn{7}{|c|}{ Parents } \\
\hline \multicolumn{7}{|l|}{ Age, $y$ (missing, $n=22$ ) } \\
\hline $17-25$ & 21.1 & 13.5 & 18.1 & 13.9 & 15.0 & \multirow{5}{*}{.04} \\
\hline $26-30$ & 30.7 & 26.7 & 25.1 & 26.7 & 27.1 & \\
\hline 31-35 & 21.5 & 27.6 & 29.7 & 25.3 & 26.3 & \\
\hline $36-40$ & 18.0 & 18.7 & 16.1 & 21.3 & 19.1 & \\
\hline$>40$ & 8.8 & 13.6 & 11.1 & 12.8 & 12.5 & \\
\hline \multicolumn{7}{|l|}{ Sex (missing, $n=20$ ) } \\
\hline Male & 3.1 & 3.4 & 2.0 & 3.0 & 3.1 & \multirow{2}{*}{.78} \\
\hline Female & 97.0 & 96.6 & 98.0 & 97.0 & 96.9 & \\
\hline
\end{tabular}

Abbreviation: BMI, body mass index.

${ }^{a}$ All sample characteristics are at the time of the child's first fall semester in Head Start. $P$ values determined by using $x^{2}$ test. the Public Health Service, the Centers for Disease Control and Prevention, or the authors' affiliated institutions. 
(continued)

Table. Characteristics of Children Enrolled in Head Start and Their Parents, Boston, Cambridge, and Somerville, Massachusetts, 2016-2019a

\begin{tabular}{|c|c|c|c|c|c|c|}
\hline \multirow[b]{2}{*}{ Characteristic } & \multicolumn{4}{|c|}{ Number of BMI measurements } & \multirow{2}{*}{$\begin{array}{c}\text { Total } \\
(\mathrm{N}=2,044)\end{array}$} & \multirow[b]{2}{*}{$P^{a}$} \\
\hline & $1(N=266)$ & $2(N=953)$ & $3(N=199)$ & $4(N=626)$ & & \\
\hline$<$ High school & 23.0 & 21.8 & 24.6 & 27.1 & 23.9 & \multirow{3}{*}{.15} \\
\hline High school graduate & 36.2 & 39.9 & 39.0 & 40.0 & 39.4 & \\
\hline$>$ High school & 40.9 & 38.3 & 36.4 & 32.9 & 36.8 & \\
\hline \multicolumn{7}{|c|}{ Family structure (missing, $n=1$ ) } \\
\hline Single parent & 69.8 & 61.8 & 65.3 & 62.9 & 63.5 & \multirow{2}{*}{.11} \\
\hline Two parents & 30.2 & 38.2 & 34.7 & 37.1 & 36.5 & \\
\hline
\end{tabular}

Abbreviation: BMI, body mass index.

${ }^{a}$ All sample characteristics are at the time of the child's first fall semester in Head Start. $P$ values determined by using $x^{2}$ test. 